SPhT/94-018; cond-mat/9402058

\title{
Entropy of Folding of the Triangular Lattice
}

\author{
P. Di Francesco \\ and \\ E. Guitter, \\ Service de Physique Théorique de Saclay *, \\ F-91191 Gif sur Yvette Cedex, France
}

\begin{abstract}
The problem of counting the different ways of folding the planar triangular lattice is shown to be equivalent to that of counting the possible 3-colorings of its bonds, a dual version of the 3-coloring problem of the hexagonal lattice solved by Baxter. The folding entropy $\log q$ per triangle is thus given by Baxter's formula $q=\sqrt{3} \Gamma(1 / 3)^{3 / 2} / 2 \pi=$ $1.2087 \ldots$.
\end{abstract}

02/94

* Laboratoire de la Direction des Sciences de la Matière du Commissariat à l'Energie Atomique. 
The link between geometrical objects like polymers or membranes and spin systems is a powerful tool of investigation. The case of phantom polymers is especially eloquent, since in its discrete form a phantom chain can be thought of as 1-dimensional Heisenberg chain, in which the spin variables stand for the directions of elementary monomers. The Heisenberg coupling is nothing but a bending energy for the (semi-flexible) polymer. Analogously, the folding of a chain is expressible as a 1-dimensional Ising model.

The 2-dimensional case is somewhat more subtle. Generically, 2-dimensional membranes can be discretized into triangulations, whose faces can support Heisenberg spin variables, now representing the direction of the local normal vector to the surface. However, one finds several universality classes of phantom membranes, depending on internal order and/or rigidity [1]. Beside the case of fluid membranes, which correspond to random triangulations, a case of special interest is that of tethered or polymerized membranes, which correspond to triangulations with fixed connectivity. In their simplest formulation, tethered membranes translate into a statistical problem on the regular triangular lattice [2]. The corresponding spin system is more involved than a simple 2-dimensional triangular Heisenberg model: indeed, as normals to a surface, the spins are not independent variables. The resulting constraints play a crucial role in favorizing an ordered phase and are responsible for the existence of a crumpling transition for tethered membranes [1-4], as opposed to the case of the usual (unconstrained) 2-dimensional Heisenberg model, always disordered.

In this letter, we address the similar, but simpler question of folding of the regular triangular lattice, the geometrical counterpart of a triangular 2-dimensional Ising model modified by additional local constraints. This model has been introduced and studied numerically in ref. [5], as a simple example in which connections between discrete and continuous models can be analyzed. It also appears to be useful for discussing analogies and differences between spin systems and geometrical objects.

After defining the folding problem and its spin formulation, we establish an equivalence between this model and the 3-coloring problem of the bonds of the triangular lattice. As a main result, this allows us to obtain the exact value of the folding entropy per triangle, which counts the different ways of folding the triangular lattice.

The Model. We define a folding as a mapping which assigns to each vertex of the triangular lattice a position in the plane, with the "metric constraint" that the distance in the plane between nearest neighbors on the lattice is maintained equal to, say, unity. Under 
such a folding, each elementary triangle of the lattice is mapped onto an equilateral triangle of the plane and each bond of the lattice serves as a hinge between its two neighboring triangles, and can be folded or not, depending on their relative position in the folded state. Up to global translations and rotations, a folded state is actually uniquely specified by the data of these elementary folds along lattice bonds.

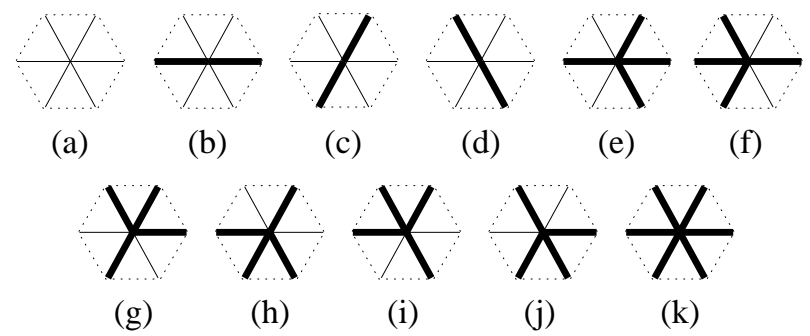

Fig. 1: The eleven local fold environments for a vertex. Folds are represented by thick lines.

It is easy to verify that, among the $2^{6}$ possible fold configurations for the six bonds surrounding a given vertex, only 11 are allowed, corresponding to actual local foldings of the hexagon surrounding the vertex. These configurations are depicted on Fig.1: configuration (a) corresponds to no fold, (b-d) to one fold of the hexagon made of two elementary bond folds, (e-j) to two folds of the hexagon (four elementary bond folds) and (k) to three folds of the hexagon ( six elementary bond folds). Note that, with the above definition, a folding does not distinguish between the different ways of folding leading to the same final state: by distinct foldings, we actually mean distinct folded state. In its original version [5], the folding problem can therefore be expressed as an eleven vertex model. Natural weights for the vertices of Fig.1 are $w_{a}=1, w_{b-d}=z, w_{e-j}=z^{2}, w_{k}=z^{3}$, which amounts to assign the weight $z$ per elementary fold (the power of $z$ in the weights $w$ is half the number of elementary folds, since a bond shared by two vertices is counted twice). Since all vertices on Fig.1 have an even number of elementary folds, these folds can be organized into folding lines without endpoints.

One can also think of foldings in terms of spin variables $\sigma_{i}= \pm$ living on the triangles, which indicate whether the $i$-th triangle faces up or down in the folded state. The spin value changes between two neighboring triangles if and only if their common bond lies on a folding line, i.e. folding lines are domain walls of the spin system. This actually leads to 


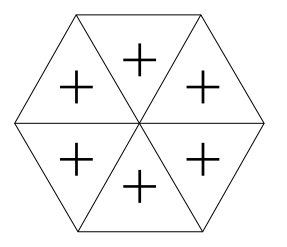

(a)

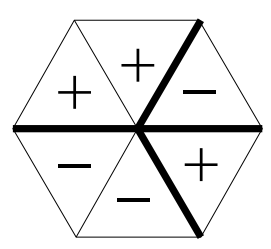

$(\mathrm{e}-\mathrm{j})$

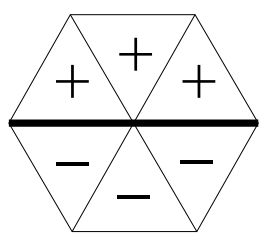

$(b-d)$

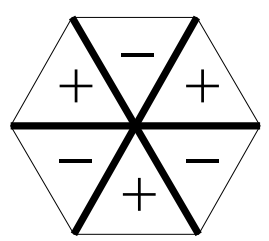

$(\mathrm{k})$

Fig. 2: Elementary folding configurations and their corresponding \pm spin configurations, up to global reversal of all spins.

two possible spin configurations per folded state, due to the degeneracy under reversal of all spins.

Conversely, arbitrary spin configurations will not in general arise from folded states. By examining the 11 possible elementary foldings (see Fig.2), we obtain a local constraint on the spin configurations around each vertex: the number of + spins around a vertex must be a multiple of 3 (i.e. 0,3 or 6 ). This local constraint turns out to be sufficient to ensure that the spin configuration actually corresponds to a folded state. The number of constrained spin configurations is indeed twice that of foldings of the hexagon $\left(\left(\begin{array}{l}6 \\ 0\end{array}\right)+\left(\begin{array}{l}6 \\ 3\end{array}\right)+\left(\begin{array}{l}6 \\ 6\end{array}\right)=\right.$ $2 \times 11$ ), as wanted. We see here, as mentioned in the introduction, an explicit realization of constraints distinguishing the geometrical folding problem from an ordinary Ising model, and ensuring that the spin variables are in fact normals to a (tethered) surface.

In this language, the above statistical weight $z$ per folded bond translates into an Ising-like interaction between nearest neighboring spins, with coupling constant $\beta J=-\frac{1}{2} \log z$. In the following we will restrict ourselves to $z=1$ and to the problem of counting the folding configurations.

Entropy. Let us consider a finite lattice with $N$ elementary triangles, and denote by $Z_{N}$ the number of its possible foldings. The entropy of folding per lattice face is defined as the thermodynamical limit

$$
s=\lim _{N \rightarrow+\infty} \frac{1}{N} \log Z_{N}=\log q
$$


In the spin language (where the number of spin configurations is clearly $Z_{\text {spin }}=2 Z_{N}$ ), $q$ is simply the average number of spin configurations per triangle, and in particular $1 \leq q \leq 2$. Such a behavior $Z_{N} \propto q^{N}$ with a non-zero value of $s$ (i.e. $q>1$ ) has been supported numerically in [5], where it is found that $q \simeq 1.21$, by use of a transfer matrix formalism for the above 11-vertex model.

The non-vanishing of $s$ is not intuitively obvious. Indeed, starting from the unfolded state with all spins + , considered as a ground state, there are no local excitations, i.e. the reversal of one spin implies that of a line of them all the way to the boundary. The reason for that is best seen on the 11-vertex version, since by drawing a vertical line across each vertex of Fig.1, one notices that whenever a fold is present on the left side of this line (cases $(\mathrm{b}-\mathrm{k})$ ), at least another one continues to the right, and conversely. Therefore, any elementary fold is part of a line crossing the entire lattice from the left to the right. In the case of the folding problem of the square lattice [4, an analogous phenomenon of non-locality results in the vanishing of the entropy $s_{\text {square }}$ (the number of folded states grows like $4^{L}$ for a square lattice of size $L \times L$, with $N=L^{2}$ faces, hence $\left.s_{\text {square }}(L)=\frac{1}{L^{2}} \log 4^{L} \underset{L \rightarrow \infty}{\longrightarrow} 0\right)$.

It is therefore interesting to directly prove the non-vanishing of $s$. This can be done by changing the definition of our ground state, namely choosing now the completely folded state, for which we pick the "antiferromagnetic" spin configuration with + on all triangles pointing up, and - on the others.

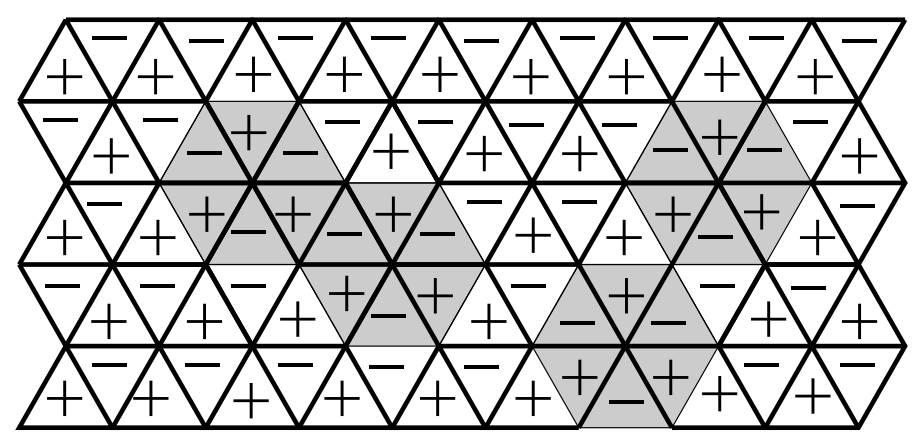

Fig. 3: Hard hexagon excitations: the spins inside the shaded hexagons are reversed with respect to the antiferromagnetic background.

Local excitations now exist, which correspond to reversing the six spins inside an elementary hexagon (see Fig.3 ). Indeed the creation of such an hexagon only affects its central and peripheral vertices, for which it exchanges spins by pairs of a + and a - spin, leaving the number of + spins surrounding these vertices unchanged. A gas of such excitations 
is also allowed, provided that two reversed hexagons do not overlap (see Fig.3 ): this is precisely the rule for the celebrated hard hexagon model, which obviously has a non-zero entropy per triangle $s_{\mathrm{hh}}>\frac{1}{6} \log 2$. This first shows the non-vanishing of the entropy of folding $s$ and moreover provides a rough lower bound $q \geq 2^{1 / 6}$. Baxter and Tsang [6] obtained the numerical estimate $2 s_{\mathrm{hh}}=.33324$, which gives a much better bound $q>1.1813$. Comparison with the numerical estimate of [5] shows that hard hexagon excitations contribute to more than 85 percent of the entropy of folding.

Equivalence with 3-colorings. Our main point in this letter is to establish that the folding problem is equivalent to a 3-coloring problem of the bonds of the triangular lattice.

We use three colors, blue (B), white (W) and red (R), ordered cyclically so that red follows white follows blue follows red. The colors can also be thought of as elements of $\mathbb{Z}_{3}=\{0,1,2\}$, defined modulo 3 .
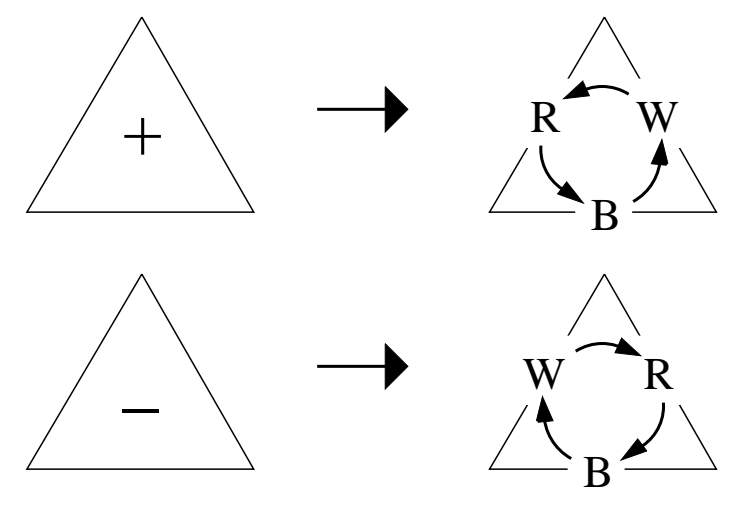

Fig. 4: Color assignments for the lattice bonds: for a $+($ resp. -$)$ spin, the colors increase (decrease) counterclockwise.

Starting from a spin configuration, we pick a bond to which we assign a color, say blue, and paint step by step all the bonds with the rule that the three bonds of any triangle have distinct colors $\mathrm{B}, \mathrm{W}, \mathrm{R}$, which are chosen in increasing (resp. decreasing) order counterclockwise, if the spin is + (resp. -), as in Fig.4. This rule fixes the colors of the bonds unambiguously, thanks to the local constraint on the spins.

To see this, it is sufficient to examine the six bonds around a vertex (see Fig.5 ): following these bonds clockwise, we see that, according to our rule, the color $c_{i+1}$ of the $(i+1)$-th bond is obtained by adding to the color $c_{i}$ of the $i$-th bond the algebraic value \pm of the crossed spin $\sigma_{i}: c_{i+1}=c_{i}+\sigma_{i} \bmod 3$. The coloring is well defined iff, after a 


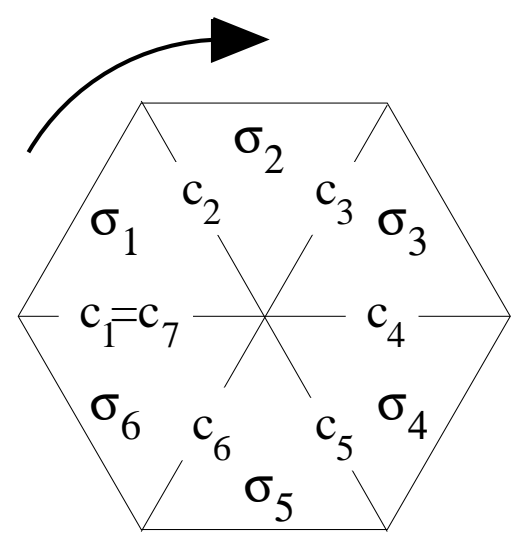

Fig. 5: The relation $c_{i+1}=c_{i}+\sigma_{i} \bmod 3$ between spin and color variables around a vertex. Coloring is unambiguous if $c_{7}=c_{1}$.

complete turn, $c_{7}=c_{1} \bmod 3$, which amounts to $\Sigma=\sigma_{1}+\sigma_{2}+\sigma_{3}+\sigma_{4}+\sigma_{5}+\sigma_{6}=0 \bmod 3$. On the other hand, $\Sigma=2 \times$ (number of + ) -6 , hence $\Sigma$ is a multiple of 3 iff the number of + spins around the vertex is a multiple of 3 , which is precisely our folding constraint on the spins.

To each spin configuration we have attached a 3-coloring of the bonds such that the three colors around each triangle are distinct. Conversely any such coloring leads to a spin configuration (spin value + if the colors increase counterclockwise, - otherwise) satisfying the folding constraint. The correspondence is 1 to 3 , due to the 3 possible choices for the color of the first bond. The number of 3-colorings is $Z_{\text {color }}=3 Z_{\text {spin }}=6 Z_{N}$.

It is interesting to notice that, in the original folding problem, the colors have a natural interpretation as the orientation of the bonds in the folded state. More precisely, let us fix a direction for the bonds of the triangular lattice, such that all the horizontal bonds point to the right, all bonds rotated by $2 \pi / 3$ point up, and all bonds rotated by $4 \pi / 3$ point down. The image of a directed bond $i$ in the folded state is a unit vector $\vec{t}_{i}$, which can only take three values: a unit vector $\vec{e}_{B}$ which fixes the (arbitrary) overall orientation of the folded state, its image $\vec{e}_{W}$ by a rotation of $2 \pi / 3$, or its image $\vec{e}_{R}$ by a rotation of $4 \pi / 3$ (with of course $\vec{e}_{B}+\vec{e}_{W}+\vec{e}_{R}=\overrightarrow{0}$ ). One can easily convince oneself that the actual value of $\vec{t}_{i}$ is simply fixed by the color $c_{i}$ of the bond $i: \vec{t}_{i}=\vec{e}_{c_{i}}$. In this respect, the 3-coloring model simply reexpresses the folding problem in terms of tangent vectors $\vec{t}_{i}$ to the surface rather than normals.

The 3-coloring problem introduced above was exactly solved by Baxter [7] in its equivalent dual form (i.e. coloring the bonds of the hexagonal lattice with three colors so that no concurrent bonds are colored alike). Using a transfer matrix method and Bethe 
ansatz techniques, Baxter obtains the following result for the entropy of 3-coloring, now reinterpreted as the exact entropy of folding $s=\log q$ :

$$
q=\prod_{n=1}^{\infty} \frac{(3 n-1)}{\sqrt{3 n(3 n-2)}}=\frac{\sqrt{\Gamma(1 / 3)}}{\Gamma(2 / 3)}=\frac{\sqrt{3}}{2 \pi} \Gamma(1 / 3)^{3 / 2}
$$

Its numerical value $q=1.208717 \ldots(s=.189560 \ldots)$ is in very good agreement with the numerical estimate of [5].

In the above, we established the equivalence between the folding problem of the triangular lattice and the exactly solvable 3-coloring problem of its bonds. This fact sheds a new light on the initial geometrical problem, and offers perspectives for further studies.

A first direction concerns the evaluation of correlation functions for geometrical quantities in the folding problem. For instance the correlation between normal vectors to the folded surface is exactly that of the spin variables, also expressible in terms of correlations of colors. Another quantity of interest is the mean square distance between two points in the folded state. We choose for instance two points $P$ and $Q$ on the same direction of the lattice, distant by $d$ lattice spacings. Using the interpretation of colors as orientations of the bonds in the folded state, the square distance between $\mathrm{P}$ and $\mathrm{Q}$ in the folded state reads

$$
r_{\mathrm{PQ}}^{2}=N_{B}^{2}+N_{W}^{2}+N_{R}^{2}-N_{B} N_{W}-N_{W} N_{R}-N_{R} N_{B}
$$

where $N_{\mathrm{B}, \mathrm{W}, \mathrm{R}}$ denote respectively the numbers of $\mathrm{B}, \mathrm{W}, \mathrm{R}$-colored bonds on the segment joining $P$ and $Q$. Based on numerical simulations [5], we expect $\left\langle r_{\mathrm{PQ}}^{2}\right\rangle \propto \log d$, the usual behavior for phantom polymerized membranes. This would amount to a color-color correlation which decreases as $1 / d^{2}$.

A second direction of study is the understanding of the effect of rigidity by introducing a weight $z=\exp -2 \beta J<1$ per fold, with the issue of a possible crumpling transition. Numerical results [5] seem to indicate that a crumpling transition takes place at $z=1$, i.e. for an infinite temperature $(\beta=0)$, with the system being in an ordered phase for any finite temperature. This agrees with the idea that a folding constraint on the spins indeed favorizes an ordered phase. In this letter, we showed that without rigidity but with the folding constraint, the model turns out to be exactly solvable. With rigidity, but no folding constraint, the model is just the triangular Ising model, exactly solvable too. These are encouraging indications that an exact solution may be found for the rigid folding problem.

We thank F. David, O. Golinelli and J.-M. Luck for useful discussions. 


\section{References}

[1] D.R. Nelson and L. Peliti, J. Phys. France 48 (1987) 1085.

[2] Y. Kantor and D.R. Nelson, Phys. Rev. Lett. 58 (1987) 2774 and Phys. Rev. A 36 (1987) 4020.

[3] M. Paczuski, M. Kardar and D.R. Nelson, Phys. Rev. Lett. 60 (1988) 2638.

[4] F. David and E. Guitter, Europhys. Lett. 5 (1988) 709.

[5] Y. Kantor and M.V. Jarić, Europhys. Lett. 11 (1990) 157.

[6] R.J. Baxter and S.K. Tsang, J. Phys. A13 Math. Gen. (1980) 1023.

[7] R.J. Baxter, J. Math. Phys. 11 (1970) 784 and J. Phys. A19 Math. Gen. (1986) 2821. 